\title{
Malignant Granular Cell Tumor with an Unusually Long Clinical Course: An Autopsy Case with Review of Literature
}

Tsuyoshi Saito ${ }^{1 *}$, Hiroyuki Mitomi ${ }^{1}$, Tomoaki Torigoe ${ }^{2}$, Tatsuya Takagi ${ }^{2}$, Yoshiyuki Suehara ${ }^{2}$, Taketo Okubo ${ }^{1,2}$, Kazuo Kaneko ${ }^{2}$ and Takashi Yao $^{1}$

${ }^{1}$ Department of Human Pathology, Juntendo University, School of Medicine, Japan

${ }^{2}$ Department of Orthopaedic Surgery, Juntendo University, School of Medicine, Japan

\begin{abstract}
We present an autopsy case of malignant granular cell tumor with an unusual clinical course. The patient had noticed a tumor on his neck 13 years prior to hospital admission. The tumor was resected and diagnosed as a malignant granular cell tumor fulfilling all 6 criteria proposed by Fanburg-Smith et al. Histologically, the tumor consisted of an extensive malignant area with adjacent small areas of benign granular cell tumor at the periphery. The patient received systemic chemotherapy and radiation, but little effect was noted. The tumor recurred 1.5 months after resection and grew rapidly. At autopsy, the tumor had metastasized to various organs, and carcinomatous lymphangiosis was present. Immunohistochemistry revealed diffuse S-100 protein expression but no expression of c-kit or EGFR and Ki-67 index in the malignant area was approximately $40 \%$. This case report demonstrates the potential of benign granular cell tumors for malignant transformation.
\end{abstract}

Keywords: Tranular cell tumor; Malignant transformation; Long clinical course

\section{Introduction}

Malignant granular cell tumor is a rare malignant soft tissue neoplasm that occurs at various sites [1-18] and accounts for $1-2 \%$ of granular cell tumors [1]. Granular cell tumors are generally divided into 3 categories according to the diagnostic criteria proposed by Fanburg-Smith et al. [1], although these criteria for malignancy are still debated among pathologists. Malignant granular cell tumors have been shown to be of Schwann cell origin $[4,5]$, although it has not yet been clearly determined whether malignant tumors arise denovo or originate from preexisting benign granular cell tumors. Furthermore, no therapeutic strategy against this tumor has yet been established, although some authors have reported that the tumor is resistant to systemic chemotherapy and that surgical resection is the best course of action [17].

We present here a case of malignant granular cell tumor with an unusual clinical course. This tumor contained small areas of benign granular cell tumor at the periphery of the primary malignant tumor, suggesting that it may have undergone malignant transformation from a benign tumor.

\section{Case Report}

A 36-year-old man had noticed a mass in the left posterior region of his neck since the age of 24 years, and the mass had been growing gradually until his first admission. He was admitted to our hospital and the tumor was resected after a biopsy that was diagnosed as a malignant tumor. The resected tumor was histologically diagnosed as a malignant granular cell tumor. The surgical margin was positive at the first treatment. The tumor recurred locally 1.5 months after the initial surgical treatment, at which time the recurrent tumor was resected and then radiation therapy (a total of 60Gy) was administered. Soon after this treatment, lung metastasis was diagnosed. The patient had also received chemotherapy consisting of high-dose ifosfamide, cisplatin, doxorubicin, and vincristine, although the therapeutic effect was only focal and the size of the tumor continued to increase gradually. Clinically, the patient was diagnosed as superior vena cava syndrome by compression of the bilateral internal jugular veins by tumors. The patient died of the disease in May 2011 at the age of 42 years.

\section{Materials and Methods}

All of the hematoxylin and eosin-stained slides from the primary and recurrent tumors and the autopsy were available for retrospective review.

\section{Immunohistochemical analysis}

Immunohistochemical staining was performed by the streptavidinbiotin method using the following antibodies: anti- $\beta$-catenin (BD transduction lab, clone 14), anti-EGFR (Leica Microsystems, UK, clone EGFR.113), anti-CD117 (Dako, Kyoto, Japan, clone rabbit polyclonal), and anti-p53 (Leica Microsystems, UK, clone PAb1801). Antigen retrieval was performed by autoclaving sections in citrate buffer.

\section{Analysis for mutations of the p53 and $\beta$-catenin genes}

Genomic DNA was extracted from the malignant granular cell tumors collected at autopsy. Exons 4-10 of the p53 gene and exon 3 of the $\beta$-catenin gene were examined for mutations by PCR followed by direct sequencing. The primer sequences used in this study have been described previously $[19,20]$.

\section{Results}

\section{Pathological findings}

The primary tumor was a subcutaneous tumor $75 \times 70 \times 42 \mathrm{~mm}$ in

*Corresponding author: Tsuyoshi Saito, M.D, Ph.D, Department of Human Pathology, Juntendo University School of Medicine, Hongo 2-1-1, Bunkyo-ku, Tokyo, Japan 113-8421, Tel: +81-3-3813-3111; Fax: +81-3-3813-3428; E-mail: tysaitou@juntendo.ac.jp

Received July 16, 2012; Accepted August 14, 2012; Published August 16, 2012

Citation: Saito T, Mitomi H, Torigoe T, Takagi T, Suehara Y, et al. (2012) Malignant Granular Cell Tumor with an Unusually Long Clinical Course: An Autopsy Case with Review of Literature. J Cancer Sci Ther 4: 260-263. doi:10.4172/1948 5956.1000152

Copyright: (c) 2012 Saito T, et al. This is an open-access article distributed under the terms of the Creative Commons Attribution License, which permits unrestricted use, distribution, and reproduction in any medium, provided the original author and source are credited. 
size that was invading into the surrounding skeletal muscles. Grossly, the tumor was grayish-white and was accompanied by focal necrosis. Histologically, the tumor cells were arranged in small nests or short trabecular patterns that occasionally involved peripheral nerve fibers. The tumor cells had eosinophilic granular cytoplasm with vesicular and prominent nucleoli. The tumor cells were tightly packed, and the nuclear to cytoplasmic ratio was high. Nuclear pleomorphism and spindling were also evident. Mitoses were observed at greater than 20/10 high-power fields (HPF), and necrosis was also noted (Figure 1A-C). Lymphocytic infiltration was also observed. Therefore, the tumor was diagnosed as a malignant granular cell tumor. The surgical margin was positive. In addition, small areas of benign granular cell tumor were observed at the periphery of the tumor, adjacent to the extensive malignant area (Figure 1D). These cells were granular, lacked nuclear atypia, had a lower nuclear to cytoplasmic ratio, and were arranged between the collagen fibers. The histology of the recurrent

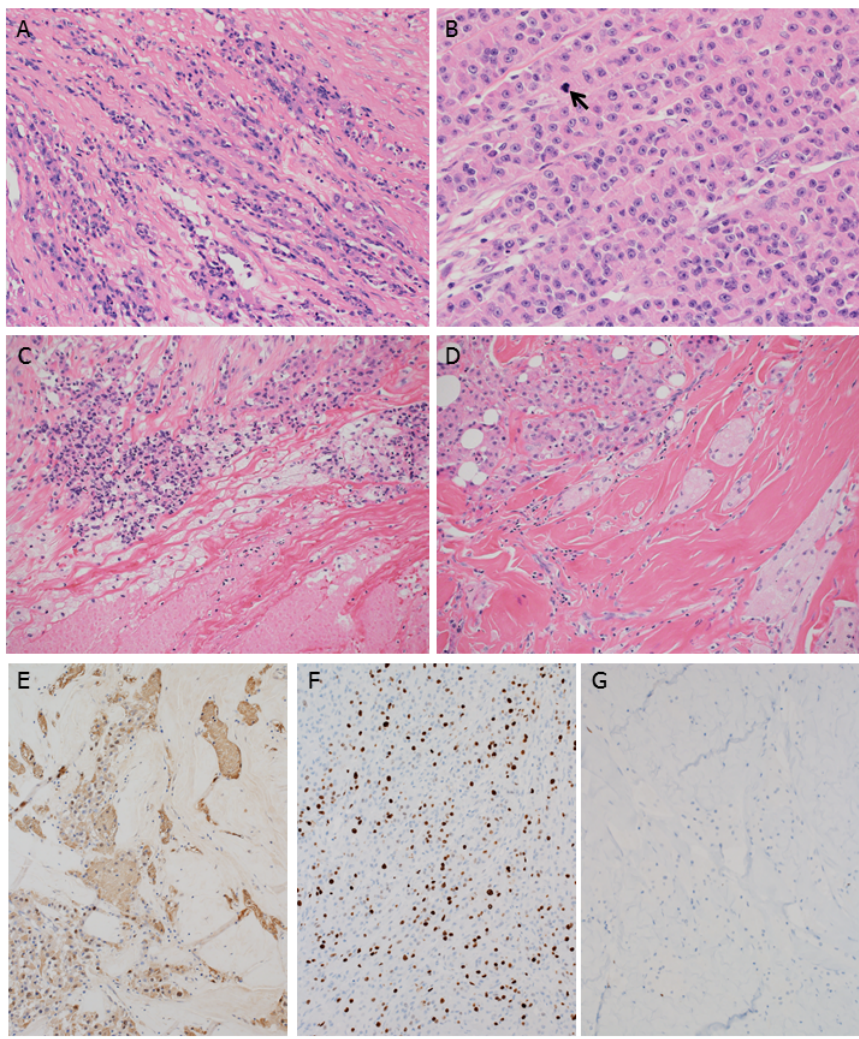

Figure 1: Histological findings of the malignant granular cell tumor A) The tumor cells were proliferating in a fascicular or trabecular fashion (H.E. staining: original magnification $\times 100$ ).

B) The tumor cells were tightly packed, and the nuclear-to-cytoplasmic ratio was high. The tumor cells had eosinophilic granular cytoplasm with vesicular and prominent nucleoli. Nuclear pleomorphism was evident. Mitoses were also seen (arrow) (H.E. staining: original magnification $\times 400)$.

C) Tumor necrosis was also noted (lower part) (H.E. staining: original magnification $\times 100$ ).

D) Small areas of benign granular cell tumor were observed at the periphery of the tumor (lower right), adjacent to the extensive malignant area (H.E. staining: original magnification $\times 100$ ).

E) Immunohistochemical expression of S-100 protein was observed at the borderline area between benign (upper right) and malignant (lower left) areas (Immunohistochemistry: original magnification $\times 100$ ).

F) $\mathrm{Ki}-67$ index in the malignant area was up to $40 \%$ in the highly malignant area (Immunohistochemistry: original magnification $x$ 100).

Ki-67 positive cell could be seldom seen in the benign area at the periphery (Immunohistochemistry: original magnification x 100). tumor was essentially similar to that of the primary tumor, although the benign granular cell tumor component was not observed.

At autopsy, the neck was quite rigid due to the multiple subcutaneous nodules. Both the left and right internal jugular veins were compressed by the tumor, which was consistent with the clinical diagnosis of superior vena cava syndrome. The tumor had metastasized to bilateral lungs, the liver, the heart, and the lumbar vertebrae in association with its dissemination to the intra-thoracic and intra-abdominal cavities. Lymph node metastasis was also noted. The tumor was composed of polymorphic tumor cells with eosinophilic granular cytoplasm, enlarged nuclei, and prominent nucleoli. Tumor necrosis was also noted. The tumor had proliferated at the serosal side of the sigmoid colon and histologically involved the muscularis propria of the colon. In the lung, in addition to forming masses, the tumor had prominently permeated the lymphatic vessels, resulting in a state of carcinomatous lymphangiosis (Figure 2A). The metastatic lesions were almost entirely composed of highly malignant granular cells (Figure 2B). The tumor cells observed in some metastatic sites showed rather less-pleomorphic histological features, namely, a lower nuclear to cytoplasmic ratio and inconspicuous nucleoli (Figure 2C). Alternatively, more and less malignant-appearing cells were seen admixed in the same metastatic regions. However, the tumor cells observed in the autopsy case were distinct from those observed in the benign granular cell component of the surgically resected specimen.

\section{Immunohistochemical findings}

The cytoplasmic granules both in malignant and benign areas of the primary tumor stained positive for S-100 protein (Figure 1E). Tumor cells observed at the autopsy was also positive for S-100 protein (Figure 2D). Immunohistochemistry for p53 was totally negative throughout the tumor. Membranous expression of $\beta$-catenin with weak cytoplasmic staining was observed; however, no nuclear staining was seen (Figure 2E). The Ki-67 index of the primary tumor was approximately up to $40 \%$ at the higher area (Figure $1 \mathrm{~F}$ ), although the tumor cells in benign area at the periphery seldom stained for Ki-67 (Figure 1G). Immunohistochemistry for c-kit was negative. The tumor cells showed no membranous staining for EGFR (Figure 2F).

\section{Genetic alteration of cancer-related genes}

No mutation of the $p 53, \beta$-catenin, or EGFR gene was detected.

\section{Discussion}

Malignant granular cell tumor is often difficult to diagnose due to its rarity. Historically, malignant granular cell tumors have been classified into 2 categories: the "histologically and clinically malignant type" and the "histologically benign, but clinically malignant type" [7]. FanburgSmith et al. [1] later proposed 6 histologic criteria for the diagnosis of atypical and malignant granular cell tumors, including necrosis, spindling, vesicular nuclei with large nucleoli, increased mitotic activity ( $>2$ mitoses/10 high-power fields at $200 \times$ magnification), high nuclear-to-cytoplasmic ratio, and pleomorphism. The primary tumor fulfilled all of the 6 above mentioned histologic criteria and was therefore diagnosed as a malignant granular cell tumor. This case could be also classified as a case of "histologically and clinically malignant type" by the former categorization scheme.

It was unusual for this tumor to be classified as a de novo primary malignant tumor considering its slowly growing character for more than 13 years without any treatment. Therefore, the clinical biological behavior of this tumor could be considered as benign, although it 


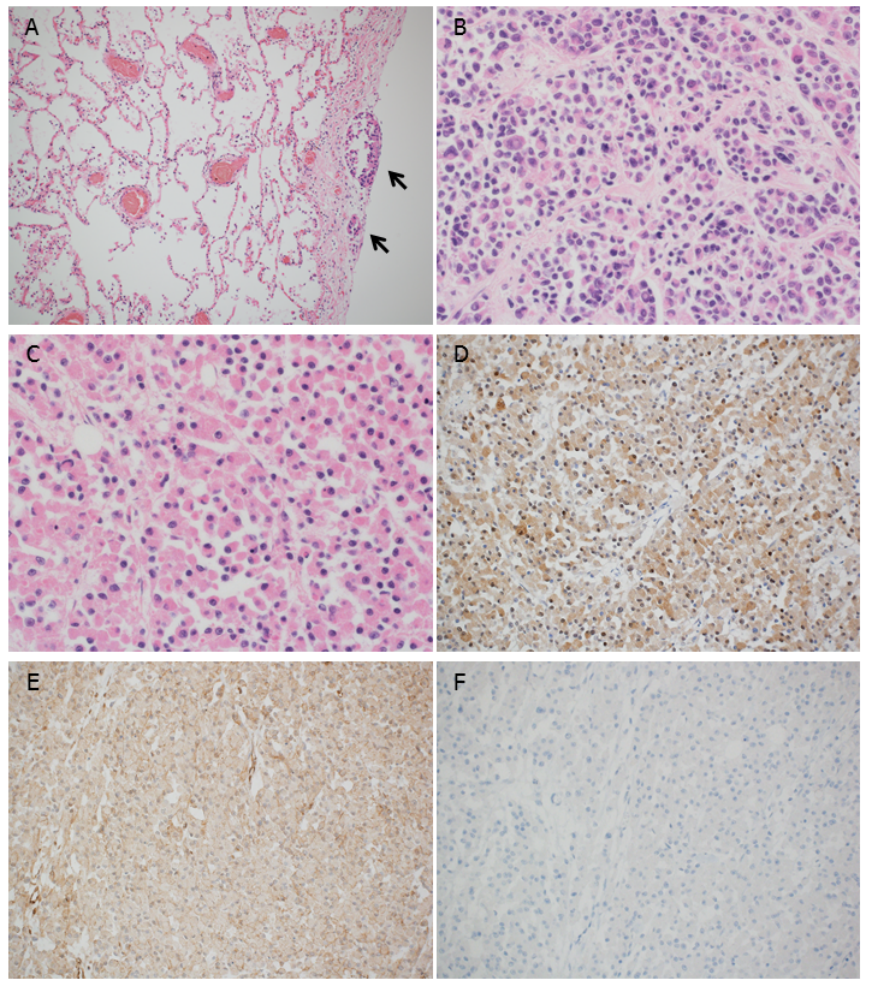

Figure 2: Histological and immunohistochemical findings in the autopsy specimens

A)Tumor cells can be seen in the lymphatic vessels below the pleura (arrows), resulting in a state of carcinomatous lymphangiosis(H.E. staining: original magnification $\times 100$ )

B) The metastatic lesions were composed almost entirely of highly malignant granular cells having high nuclear to cytoplasmic ratio and hyperchromatic enlarged nuclei (H.E. staining: original magnification x 200).

C) The tumor cells observed in some metastatic regions showed rather lesspleomorphic histological features, including lower nuclear-to-cytoplasmic ratios and inconspicuous nucleoli (H.E. staining: original magnification $\times 200$ ). D) The cytoplasmic granules stained positive for S-100 protein (Immunohistochemistry: original magnification $\times 100$ ).

E) Membranous expression of $\beta$-catenin with weak cytoplasmic staining was observed; however, no nuclear staining was seen (Immunohistochemistry: original magnification $\times 100$ )

F) The tumor cells showed no membranous staining for EGFR (Immunohistochemistry: original magnification x 100).

grew rapidly after the initial surgical resection. A quite similar case has been recently reported, where 10 years without rapid growth has been passed and tumor has started to grow rapidly after the surgery, although the primary tumor has been diagnosed as an atypical granular cell tumor by fulfilling 2 of the 6 aforementioned criteria [18]. The Ki-67 index of the atypical granular cell tumor has been described as approximately $10 \%$ [18]. The recurrent tumor has been described to have transformed to a malignant granular cell tumor [18]. Malignant transformation has been also reported in a case of benign granular cell tumor [3]. Therefore, in consideration of the possibility that the tumor had undergone malignant transformation, the tumor was microscopically examined throughout the initially resected specimen (a total of 52 slides). Small areas of apparently histologically benign granular cell tumor were observed adjacent to the highly malignant areas, mainly at the periphery of the tumor. Alternatively, a transition of the sarcomatous regions to a benign appearance in a malignant granular cell tumor has been reported by Cadotte [8], though the histology has not been well-demonstrated in this case. Furthermore, the lesion of atypical granular cell tumor has been demonstrated to coexist with malignant granular cell tumor [21]. In this case, the metastatic lesions observed at the autopsy were, with some exceptions, composed almost entirely of highly malignant granular cells with necrosis. The tumor cells observed in some metastatic regions showed histologically rather less pleomorphic features compared with those in the highly malignant area; these tumor cells had lower nuclear-to-cytoplasmic ratios and inconspicuous nucleoli, although occasional binuclear cells were encountered. The less-pleomorphic areas observed in this case could be classified as atypical granular cell tumor, as they still fulfilled 2 of the 6 criteria, namely, vesicular nuclei with large nucleoli and pleomorphism. A case of malignant granular cell tumor in which the metastatic lesion was composed of rather benign-appearing tumor cells has also been reported [4]. Malignant granular cell tumors could therefore be suggested to show a relatively wide range of histology, encompassing apparently malignant features and somewhat benignappearing features. However, no purely benign-appearing area could be detected at autopsy. These findings may suggest that this is a second case in which a benign tumor transformed into a malignant granular cell tumor during a long clinical course.

In one study, 14 of 25 (56\%) malignant tumors had Ki-67 immunostaining in $10-50 \%$ of the cell population [1]. In this case, approximately $40 \%$ of the tumors were positive for Ki-67 in malignant area, in line with the previous finding. However, positive tumor cells were seldom seen in benign area at the periphery. p53 immunostaining was also reported in more than $50 \%$ of the cell population in 17 of 25 (68\%) malignant granular cell tumors [1]. Furthermore, p53 expression has been reported in several cases of malignant granular cell tumors [17]. However, no p53-positive cells or p53 mutations were detected in this case. These findings strongly suggest that it is very difficult to diagnose malignant granular cell tumor by immunohistochemical techniques alone.

$\beta$-catenin is a multi-functional protein that plays an important role in maintaining cell-cell adhesion as well as a downstream effector of the Wnt-signaling cascade. Expression and mutations of $\beta$-catenin in soft tissue sarcomas has also been described [20], though $\beta$-catenin expression in granular cell tumors including malignant counterpart has not yet reported so far. Furthermore, possibilities of molecular therapy targeting of Wnt signaling has been demonstrated in a certain type of malignancies [22]. Therefore, expression and mutational status of $\beta$-catenin in this tumor was examined. Definite membranous expression of $\beta$-catenin was observed without cytoplasmic and nuclear staining, suggesting that activation of Wnt signaling has less importance in this tumor.

The early relapse of the tumor in this case may have been due in part to the positive surgical margins of the initial surgery. Alternatively, the surgery may have activated the tumor growth. At all sites of malignant granular cell tumors, the overall survival is poor. The mortality rate within 3 years has been described to be close to $60 \%$ [23]. No treatment for malignant granular cell tumors has yet been well established, and no evidence for the efficacy of chemotherapy against malignant granular cell tumor has been shown to date [17]. This tumor also showed resistance to chemotherapy and radiation therapy. In the hope of identifying molecular therapeutic targets in malignant granular cell tumors, immunohistochemistry for c-kit and EGFR, for which appropriate tyrosine kinase inhibitors are available, was performed. However, the expression statuses of these receptor tyrosine kinases were totally negative, consistent with the findings of the mutation 
Citation: Saito T, Mitomi H, Torigoe T, Takagi T, Suehara Y, et al. (2012) Malignant Granular Cell Tumor with an Unusually Long Clinical Course: An Autopsy Case with Review of Literature. J Cancer Sci Ther 4: 260-263. doi:10.4172/1948-5956.1000152

analysis. Further information regarding the response of this tumor to various therapeutic agents must be gathered in order to develop an effective therapeutic strategy.

\section{Conclusions}

This case report demonstrates the potential of benign granular cell tumors for malignant transformation.

\section{Acknowledgement}

This work was supported in part by Grants-in-Aid for General Scientific Research from the Ministry of Education, Science, Sports, and Culture (\#23590434 to Tsuyoshi Saito and \#24590429 to Hiroyuki Mitomi), Tokyo, Japan.

\section{References}

1. Fanburg-Smith JC, Meis-Kindblom JM, Fante R, Kindblom LG (1998) Malignant granular cell tumor of soft tissue: diagnostic criteria and clinicopathologic correlation. Am J Surg Pathol 22: 779-794.

2. Schoedel KE, Bastacky S, Silverman A (1998) An S-100 negative granular cell tumor with malignant potential: report of a case. J Am Acad Dermatol 39: 894898.

3. Gomard-Mennesson E, Isaac S, Freymond N, Guibert B, Pacheco Y, et al. (2007) Pulmonary metastases from Abrikossoff's tumour. Transformation capability of a benign granular cell tumor. Rev Mal Respir 24: 900-904.

4. Usui M, Ishii S, Yamawaki S, Sasaki T, Minami A (1977) Malignant granular cell tumor of the radial nerve: an autopsy observation with electron microscopic and tissue culture studies. Cancer 39: 1547-1555.

5. Shimamura K, Osamura RY, Ueyama Y, Hata J, Tamaoki N, et al. (1984) Malignant granular cell tumor of the right sciatic nerve. Report of an autopsy case with electron microscopic, immunohistochemical, and enzyme histochemical studies. Cancer 53: 524-529.

6. Di Tommaso L, Magrini E, Consales A, Poppi M, Pasquinelli G, et al. (2002) Malignant granular cell tumor of the lateral femoral cutaneous nerve: report of a case with cytogenetic analysis. Human Pathol 33: 1237-1240.

7. Gamboa LG (1955) Malignant granular-cell myoblastoma. AMA Arch Patho 60: $663-668$

8. Cadotte M (1974) Malignant granular-cell myoblastoma. Cancer 33: 14171422.

9. Chelly I, Bellil K, Mekni A, Bellil S, Belhadjsalah M, et al. (2005) Malignant granular cell tumor of the abdominal wall. Pathologica 97: 130-132.

10. Nasser H, Danforth RD Jr, Sunbuli M, Dimitrijevic O (2010) Malignant granular cell tumor: case report with a novel karyotype and review of the literature. Ann Diagn Pathol 14: 273-278.

11. Rekhi B, Jambhekar NA (2010) Morphologic spectrum, immunohistochemica analysis, and clinical features of a series of granular cell tumors of soft tissues: a study from a tertiary referral cancer center. Ann Diagn Pathol 14: 162-167.

12. Hwang JS, Beebe KS, Rojas J, Peters SR (2011) Malignant granular cell tumor of the thigh. Orthopedics 34: e428-431.

13. Suzuki S, Maeda S, Sasajima K, Yoshida H, Yokoyama T, et al. (2010) Malignant granular cell tumor in the gluteal region with unusual pathologic features. Int Surg 95: 360-365.

14. Parayno PP, August CZ (1996) Malignant granular cell tumor. Report of a case with DNA ploidy analysis. Arch Pathol Lab Med 120: 296-300.

15. Wieczorek TJ, Krane JF, Domanski HA, Akerman M, Carlen B, et al. (2001) Cytologic findings in granular cell tumors, with emphasis on the diagnosis of malignant granular cell tumor by fine-needle aspiration biopsy. Cancer 93: 398408.

16. Jiang M, Anderson T, Nwogu C, Tan D (2003) Pulmonary malignant granular cell tumor. World J Surg Oncol 1: 22.

17. Schmidt O, Fleckenstein GH, Gunawan B, Fuzesi L, Emons G (2003) Recurrence and rapid metastasis formation of a granular cell tumor of the vulva. Eur J Obstet Gynecol Reprod Biol 106: 219-221.

18. Akahane K, Kato K, Ogiso S, Sakaguchi K, Hashimoto M, et al. (2012) Malignant granular cell tumor of the breast: case report and literature review. Breast Cancer.

19. Saito T, Mitomi H, Suehara Y, Okubo T, Torigoe T, et al. (2011) A case of de novo secondary malignant giant-cell tumor of bone with loss of heterozygosity of $p 53$ gene that transformed within a short-term follow-up. Pathol Res Pract 207: 664-669.

20. Saito T, Oda Y, Yamamoto H, Kawaguchi K, Tanaka K, et al. (2006) Nuclea beta-catenin correlates with cyclin D1 expression in spindle and pleomorphic sarcomas but not in synovial sarcoma. Human Pathol 37: 689-697.

21. Mahoney A, Garg A, Wolpowitz D, Mahalingam M (2010) Atypical granular cell tumor-apropos of a case with indeterminate malignant potential. Am J Dermatopathol 32: 370-373.

22. Jarde T, Evans RJ, McQuillan KL, Parry L, Feng GJ, et al. (2012) In vivo and in vitro models for the therapeutic targeting of Wnt signaling using a Tet-O $\Delta \mathrm{N} 89 \beta$ catenin system. Oncogene.

23. Argenyi ZB (2006) Granular cell tumor in LeBoit PE, Burg G, Weedon D, Sarasin A, (Eds). Pathology and genetics of skin tumors. Lyon: IARC press. 